\title{
A Population-based Investigation of the Autoantibody Profile in Mothers of Children with Atrioventricular Block
}

S Salomonsson, A Ambrosi, E Fernlund, E Theander, A Ohman, A Rydberg, Thomas Skogh, S Wallberg-Jonsson, A Elfving, M Mellander, O Winqvist,

F Gadler and M Wahren-Herlenius

\section{Linköping University Pre Print}

N.B.: When citing this work, cite the original article.

This is the pre-reviewed version of the following article:

S Salomonsson, A Ambrosi, E Fernlund, E Theander, A Ohman, A Rydberg, Thomas Skogh, S Wallberg-Jonsson, A Elfving, M Mellander, O Winqvist, F Gadler and M WahrenHerlenius, A Population-based Investigation of the Autoantibody Profile in Mothers of Children with Atrioventricular Block, 2011, Scandinavian Journal of Immunology, (74), 5, 511-517.

which has been published in final form at:

http://dx.doi.org/10.1111/j.1365-3083.2011.02610.x

Copyright: Blackwell Publishing http://www.blackwellpublishing.com/

Postprint available at: Linköping University Electronic Press http://urn.kb.se/resolve?urn=urn:nbn:se:liu:diva-72019 


\section{A population-based investigation of the autoantibody profile in mothers of children with atrioventricular block}

Short title: Autoantibody profile in mothers of children with heart block

Stina Salomonsson ${ }^{1}$, Vijole Dzikaite ${ }^{1}$, Elisabeth Zeffer ${ }^{1}$, Håkan Eliasson ${ }^{2}$, Aurelie Ambrosi $^{1}$, Gunnar Bergman ${ }^{2}$, Eva Fernlund ${ }^{3}$, Elke Theander ${ }^{4}$, Annika Öhman $^{5}$, Annika Rydberg $^{6}$, Thomas Skogh $^{7}$, Solveig Wållberg-Jonsson ${ }^{8}$, Åse Elfving $^{1}$, Michael Fored ${ }^{1}$, Anders Ekbom ${ }^{1}$, Ulla Lundström ${ }^{9}$, Mats Mellander ${ }^{9}$, Ola Winqvist ${ }^{1}$, Sven-Erik Sonesson $^{2}$, Fredrik Gadler ${ }^{1}$, Anders Jonzon ${ }^{5}$, Marie Wahren-Herlenius ${ }^{1}$

${ }^{1}$ Department of Medicine Solna, ${ }^{2}$ Department of Women and Child Health, Karolinska Institutet, Stockholm, ${ }^{3}$ Department of Pediatric Cardiology, Skane University Hospital, Lund, ${ }^{4}$ Rheumatology Department, Skåne University Hospital, Malmö, ${ }^{5}$ Department of Women's and Children's Health, Section for Pediatrics, Uppsala University, Uppsala

${ }^{6}$ Department of Clinical Sciences, Paediatrics, Umeå University, Umeå,

${ }^{7}$ Rheumatology/AIR, Clinical and Experimental Medicine, Linköping University, Linköping, ${ }^{8}$ Department of Rheumatology, Umeå University, Umeå, ${ }^{9}$ Department of Pediatric Cardiology, The Queen Silvia Children`s Hospital, Sahlgrenska University Hospital, Göteborg

\section{Corresponding author:}

Marie Wahren-Herlenius 
Rheumatology Unit, Department of Medicine Solna

Karolinska Institutet, 17176 Stockholm, Sweden

Phone: +46-8-51773431, Fax: +46-8-51775562

Email: marie.wahren@ki.se

\author{
Abstract word count: 231 \\ Manuscript word count: 2838 \\ Scientific heading: Clinical Immunology
}




\section{ABSTRACT}

The objective of the study was to investigate the antigen specificity and occurrence of individual autoantibodies in mothers of children diagnosed with atrioventricular (AV) block in a nation-wide setting. Patients with AV block detected before 15 years of age were identified using national quality registries as well as a network of pediatric and adult cardiologists and rheumatologists at the six university hospitals in Sweden. Patients with gross heart malformations, surgically or infectiously induced blocks were excluded. Blood samples were obtained from the mothers and maternal autoantibody profile, including the occurrence of antibodies against Ro52, Ro60, La, SmB, SmD, RNP-70k, RNP-A, RNP-C, CENP-C, Scl-70, Jo-1, ribosomal RNP and histones was investigated in 193 mothers of children with AV block by immunoblotting and ELISA. Autoantibody reactivity was detected in $48 \%$ (93/193) of the mothers of children with AV block. In autoantibody-positive mothers, the vast majority, 95\% (88/93), had antibodies against Ro52, while 63\% (59/93) had autoantibodies to Ro60 and 58\% (54/93) had autoantibodies to La. In addition, 13\% (12/93) of the autoantibody-positive mothers had antibodies to other investigated antigens besides Ro52, Ro60 and La, and of these antihistone antibodies were most commonly represented, detected in 8\% (7/93) of the mothers. In conclusion, this Swedish population-based study confirm that maternal autoantibodies may associate with heart block in the child. Further, our data demonstrate a dominant role of Ro52 antibodies in association with AV block. 


\section{INTRODUCTION}

Congenital complete heart block without anatomical malformations is a rare disease with an incidence of $1 / 15,000-20,000$ in the general population [1], while the prevalence of complete congenital heart block in pregnancies of women with anti-Ro/SSA antibodies is approximately $2 \%$ [2]. These women are commonly diagnosed with systemic lupus erythematosus (SLE) or Sjögren's syndrome, but may also be asymptomatic. The fetal atrioventricular (AV) block usually develops during the 18-24th week of gestation after transfer of the maternal antibodies across the placenta, but can also be diagnosed later [35]. Following reports of increased incidence of sudden deaths in asymptomatic patients with AV block [6], a pacemaker is now commonly implanted at an early age.

We and others have shown that early treatment of a second degree AV block with high-dose fluorinated steroids may prevent progression of, or even revert the block, decreasing fetal morbidity and mortality [7-10]. A complete third-degree block is however regarded permanent, stressing the importance to find diagnostic and prognostic markers that will identify the high risk pregnancies and enable timely treatment.

Autoantibodies to the Ro52, Ro60 and La proteins have all been associated with development of congenital heart block (reviewed in Salomonsson et al 2010 [11]). Several studies highlight a dominant role of antibodies toward Ro52 in the cases of heart block [2, 12-15]. After reports that maternal antibodies to the amino acid (aa) 200-239 (p200) of the Ro52 protein bind to the cell surface of cardiomyocytes and induce apoptosis of affected cells by dysregulating the intracellular calcium homeostasis [16, 17], these specific Ro52/p200 antibodies were suggested as a serologic marker for an 
increased risk of having a child with congenital heart block [14, 18]. Anti-histone antibodies have also been implicated in development of congenital heart block [13], but other common autoantibodies in systemic rheumatic disease have been less systematically analyzed. Knowledge of the occurrence of such specificities may be important to improve diagnostics or better understand the pathogenicity of the condition. Given the rarity of the condition and to decrease selection bias, we performed a nation-wide study in Sweden to investigate the prevalence of autoantibodies in mothers of children diagnosed with AV block before 15 years of age in a population-based setting. Patients with heart block were identified using national registries as well as a network of clinical investigators at the six university hospitals in Sweden. The mothers of patients identified were asked to participate in the study by donating a blood sample, and the autoantibody profile to 13 autoantigens, including occurrence of antibodies against Ro52, Ro60 and La, was analyzed. 


\section{MATERIALS AND METHODS}

\section{Identification of patients}

Patients with AV block detected before 15 years of age were identified combining three different strategies; (1) searching the Swedish national patient register, (2) searching the national Swedish implantable cardioverter defibrillator (ICD) and pacemaker register, and (3) by local clinical pediatric and adult cardiology registers and rheumatology registers at the six university hospitals in Sweden, Figure 1.

For identification of patients in the Swedish national patient registers a search using the International Classification of Diagnosis (ICD-8, -9 and -10) codes for AV block II and III was performed in the inpatient and outpatient registers. The inpatient register contains information on medical discharge diagnoses and was established in 1964, with complete nationwide coverage since 1987. The outpatient register contains $>90 \%$ of all medical specialist visits in Sweden since 2002. This strategy resulted in a set of 9190 entries from 808 patients.

A separate search was performed in the national Swedish ICD and Pacemaker register. The register was established in 1989, and all 44 centers in Sweden where pacemaker implants are performed are linked to the register, giving a coverage of close to 100\% (Annual statistical report 2009, http://www.pacemakerregistret.se/icdpmr/annualReport/2009/annualReport_2009.pdf). Patients receiving their first pacemaker implant before 15 years of age and with the diagnosis AV block were selected, which resulted in a set of 263 patients. 
The third approach taken to identify patients with heart block was through identification in local hospital registers of cases or family members seen by cardiologists or rheumatologists at the six university hospitals in Sweden. Two hundred and fifty-nine patients were identified at the Karolinska University Hospital, the Uppsala University Hospital, the Umeå University hospital, the Skåne University Hospital Malmö/Lund, the Linköping University Hospital and the Sahlgrenska University Hospital.

Overlaps were identified and all unique cases were cross-matched to the Swedish malformation register to exclude patients born with gross heart malformations. In addition, a manual review of all registered diagnosis codes for all individuals was performed to ensure exclusion of patients with significant heart malformations as well as patients with post-operative AV block and patients with infection-induced heart block. After excluding these, 338 individual patients, born during 1914-2010, with AV block diagnosed before 15 years of age remained. Contact was established with 314 of the 338 index cases (93\%). The majority of contacted patients chose to participate in the study $(279 / 314,89 \%)$. The mothers of 215 of the participating 279 index cases were alive. To investigate maternal autoimmune-associated serology, the live mothers with children affected by AV block II/III were contacted and asked to participate in the study and to donate a blood sample. Plasma samples were obtained from 193 mothers and analyzed for autoantibody reactivity. $\underline{\text { Clinical information on maternal disease was not collected. }}$ The study was approved by the Regional Ethical Committee Stockholm, Karolinska Institutet, and all participants gave written informed consent. 


\section{Autoantibody analyses}

Antibodies against Ro52, Ro60, La, SmB, SmD, RNP-70k, RNP-A, RNP-C, CENP-C, Topo-I/Scl-70, Jo-1/HRS, ribosomal RNP and histones were detected through a commercial line blot analysis (Inno-Lia ${ }^{\mathrm{TM}}$ ANA Update, Innogenetics, Cambereley, United Kingdom), according to the manufacturer's instructions.

Autoantibodies to Ro52 were also analyzed by ELISA as previously described [14]. In short, medium-binding 96-well plates (Nunc, Odense, Denmark) were coated with $1 \mu \mathrm{g}$ per well of recombinant full-length Ro52 protein diluted in carbonate buffer, $\mathrm{pH}$ 9.6. Plates were blocked with phosphate buffered saline (PBS)/0.05\% Tween $/ 5 \%$ milk powder, and sera were tested at a dilution of 1:500 in PBS/0.05\% Tween/1\% milk powder. Bound antibodies were detected by affinity-purified, alkaline phosphataseconjugated anti-human IgG, IgA or IgM antibodies (Dakopatts, Glostrup, Denmark). Phosphatase substrate tablets (Sigma, St. Louis, MO) dissolved in diethanolamine buffer, pH 9.6 were used as substrate. The absorbance was measured at $405 \mathrm{~nm}$. All steps were performed at room temperature except coating, which was performed at $4^{\circ} \mathrm{C}$. For the $\mathrm{IgG}$ analyses, sera from ten healthy subjects lacking anti-Ro52 antibodies were used as controls and the cut-off for a positive autoantibody test set at the mean of the healthy controls +2SD. For IgA and IgM analyses, sera from 15 age- and sex-matched individuals were used as controls and cut-off set at the mean of controls +5SD.

Antibodies to the p200 epitope of Ro52, encompassing amino acid residues 200239 of the Ro52 protein, were detected using the Wieslab® SS-A p200 ELISA kit (Eurodiagnostica, Malmö, Sweden) according to the manufacturer's instructions (www.eurodiagnostica.com). All analyses were performed at the Department of 
Medicine, Karolinska Institutet during the same time period. The Inno-Lia ANA Update and SS-A p200 ELISA were performed at the Clinical Immunology Unit and the Ro52 IgG, IgA and IgM ELISA at the Rheumatology Unit.

\section{Statistical analysis}

The correlation between p200 antibody levels and i) duration from delivery of a child with AV block or ii) maternal age at the time of blood sampling for this study was analyzed by the two-tailed Spearman correlation test using the computer software program GraphPad Prism version 5.01. A p-value $<0.05$ was considered significant. 


\section{RESULTS}

\section{Identification of index cases and collection of blood samples}

Patients in Sweden with AV block, without major cardiac malformations and diagnosed before 15 years of age were identified using three different strategies, Figure 1. Mothers were identified via the index cases and contacted. Blood samples from 193 mothers were collected and analyzed.

\section{Maternal autoantibody profiles are dominated by anti-Ro52 antibodies}

To investigate the spectrum of autoantibodies carried by mothers of children with AV block, plasma derived from the blood samples was tested in a line blot assaying IgG
autoantibodies to Ro52, Ro60, La, histones, SmB, SmD, RNP-70k, RNP-A, RNP-C,
CENP-C, Topo-I/Scl-70, Jo-1/HRS and ribosomal RNP. In $52 \%(\mathrm{n}=100 / 193)$ of the
mothers no autoantibody reactivity was detected, while $48 \%(\mathrm{n}=93)$ of the mothers
carried circulating autoantibodies, Figure 2 . In autoantibody-positive mothers, the vast
majority, 95\% (88/93), had antibodies to Ro52. Antibodies to Ro60 were detected in $63 \%$ (59/93) of the mothers, and 58\% (54/93) had autoantibodies to La, Figure 3 and Table 1. In four out of the five maternal sera that lacked Ro52 antibodies but had an autoantibodypositive profile, antibodies to Ro60 and/or La were detected. In one of the five mothers antibodies to histones were detected. Of all the autoantibody-positive mothers $13 \%$ ( $n=12 / 93$ ) had antibodies to other investigated antigens besides Ro52, Ro60 and La. Of these, anti-histone antibodies were the most commonly represented and found in $8 \%$ (7/93) of the mothers, Figure 3A. 
Autoantibodies to $\operatorname{Ro52}$ of the $\operatorname{IgA}$ and $\operatorname{IgM}$ isotypes were also investigated in all maternal sera. Nearly half of the investigated IgG anti-Ro52 positive maternal cohort, 47\% (41/88), displayed IgA anti-Ro52 antibodies, while 30\% (26/88) had IgM anti-Ro52 antibodies, Figure 3B. In addition, two maternal sera negative for IgG anti-Ro52 antibodies contained IgA anti-Ro52 antibodies, although at levels close to the cut-off value.

\section{Antibodies to the p200 epitope in anti-Ro52 positive sera}

Antibodies to amino acid 200-239 (p200) have been suggested to be specifically associated with development of heart block, and we therefore investigated p200-specific antibody levels in anti-Ro52 positive mothers. Antibodies toward p200 were detected in $60 \%(53 / 88)$ of sera from Ro52-positive mothers. Samples were considered positive if the arbitrary p200 level was >0.7 (according to manufacturer's instructions). Levels of antip200 antibodies in all Ro52 positive maternal sera ranged from 0.17 to 4.30 units, $1.4 \pm$ 1.2 (mean, SD), Figure 4.

Both the age of the mothers at the time of sampling and the time elapsed between giving birth to a child with AV block and blood sampling differed between the mothers. These factors could potentially affect the p200 antibody levels and an analysis of the correlation between p200 antibody levels and time elapsed since giving birth to a child with AV block as well as maternal age was therefore performed, Figure 5. A statistically significant correlation was found between the time elapsed since giving birth to a child with AV block and anti-p200 antibody levels (Figure 5A), with lower antibody levels in mothers with a longer time lapse between delivery and blood sampling $(r=-0.40$, 
$\mathrm{p}<0.0001)$. In addition, a significant correlation between maternal age at blood sampling and p200 antibody levels was detected $(r=-0.33, \mathrm{p}=0.0016)$, Figure 5B. 


\section{DISCUSSION}

Congenital heart block is the most serious complication of the neonatal lupus syndrome, and is thought to result from an inflammatory reaction in the fetal heart tissue associated with maternal autoantibodies $[16,19]$. The inflammatory reaction may be mild and reversible (AV block I), or more advanced (AV block II) and in such cases commonly leads to fibrosis and calcification replacing the conductive tissue, clinically presenting as a complete irreversible block (AV block III) [20]. Treatment of a second degree AV block with fluorinated steroids has been reported to prevent the initiated block from proceeding to a more severe state, making it important to identify risk pregnancies to allow timely treatment [7-9].

In the present study, we identified patients with AV block using a nation-wide register-based strategy, and examined the maternal autoimmune serology associated with AV block detected before 15 years of age in the children. We found that approximately half of the mothers investigated (48\%) carried autoantibodies, and of these mothers the vast majority (95\%) had antibodies against the Ro52 component of the SSA antigen. A major proportion of these sera contained autoantibodies recognizing the p200 epitope of the Ro52 protein, an epitope that has been linked to the development of $\mathrm{CHB}$ in animal models [16].

Notably, in half of the patients $(52 \%)$ diagnosed with AV block before 15 years of age in this Swedish cohort, an association with maternal autoantibodies could not be confirmed. We used strategies aimed at identifying all cases on a nation-wide basis, but a potential bias in the inclusion exists as the strategies have different coverage over 
different time periods. Further, the present study is based on analysis of sera from live mothers, and a higher mortality can be expected for mothers of the autoantibody-positive group which will include mothers diagnosed with SLE, having a shorter life expectancy. This could generate a falsely high number of autoantibody negative pregnancies with AV block, especially for older cases before introduction of more efficient therapy for SLE. Our finding that approximately half of the cases of AV block before 15 years of age associate with maternal autoantibodies is however in line with the relationship regarding autoimmune serology and heart block previously reported by Villain and colleagues in France who described that $49.5 \%$ of the mothers carried autoantibodies and $50.5 \%$ of $\underline{\text { mothers had no detectable autoantibodies [21]. Also Julkunen and co-workers found a }}$ substantia proportion, $41 \%$, of non-autoantibody carrying mothers in identification of early-onset AV block in a Finnish hospital-register based study [22]. Different pathogenic mechanisms in the absence of autoantibodies have been suggested, including mono- and polygenetic associations, and these conditions may have a less severe prognosis than heart block associated with maternal autoantibodies [21-23].

The finding of a strong association between heart block and autoantibodies, and specifically antibodies against Ro52/SSA, confirms previous results reported by us and others $[2,14,16,18,22,24-28]$. Earlier reports describe an antibody profile in mothers of children with congenial heart block with a prevalent, but not unique, anti-Ro52 antibody response [14, 21, 26-30]. Previous studies have suggested that antibodies to Ro60 and La have a minor role in predicting the fetal clinical outcome in Ro and La positive mothers $[14,26,31]$, though maternal La antibodies in addition to Ro/SSA antibodies have been suggested to increase the risk for heart block in the child from $2 \%$ to $3.1 \%$ [32]. Five of 
the mothers in this report having children with heart block did not have antibodies to Ro52. However, three of the five Ro52 negative mothers would have been detected as SSA positive as they were anti-Ro60 autoantibody positive. One autoantibody-positive mother with no reactivity against the Ro52 protein demonstrated antibodies against histone only, a rare finding associated with development of heart block reported previously [13].

An association between congenital heart block and maternal antibodies to the p200 epitope (amino acid 200-239) of the Ro52 protein has previously been demonstrated $[16,18]$. The negative correlation observed in this study between the level of anti-p200 antibodies and time elapsed from delivery implies that this may partly account for the low levels of p200 specific antibodies in some of the mothers.

In conclusion, our data from the Swedish population confirm that maternal autoantibodies are associated with complete heart block in half of the cases detected before 15 years of age, and demonstrate that Ro52 autoantibodies are the most commonly occurring autoantibodies associated with the development of heart block. 


\section{ACKNOWLEDGEMENTS}

We gratefully acknowledge Paul Blomquist for excellent insights and guidance regarding the epidemiological part of this work, and Eva Jemseby and Gull-Britt Almgren for excellent technical assistance in blood sample processing.

Financial support for this study was obtained from KIRCNET (Karolinska Institutet Circulation and Respiratory Research Network), the Magn Bergvalls Foundation, the Jerringfoundation, Stiftelsen Samariten, the Karolinska Institute, The Royal Swedish Academy of Sciences, the Swedish Research Council, the Göran Gustafsson Foundation, the Torsten and Ragnar Söderberg Foundation, the King Gustaf the V:th 80-year foundation, the Swedish Foundation for Strategic Research, the HeartLung Foundation and the Swedish Rheumatism Association. 


\section{REFERENCES}

1. Michaelsson M, Engle MA. Congenital complete heart block: an international study of the natural history. Cardiovasc Clin 1972;4:85-101.

2. Brucato A, Frassi M, Franceschini F, et al. Risk of congenital complete heart block in newborns of mothers with anti-Ro/SSA antibodies detected by counterimmunoelectrophoresis: a prospective study of 100 women. Arthritis Rheum $2001 ; 44: 1832-5$

3. Buyon JP, Hiebert R, Copel J, et al. Autoimmune-associated congenital heart block: demographics, mortality, morbidity and recurrence rates obtained from a national neonatal lupus registry. J Am Coll Cardiol 1998;31:1658-66.

4. Sonesson SE. Diagnosing foetal atrioventricular heart blocks. Scand J Immunol 2010;72:205-12.

5. Brucato A, Previtali E, Ramoni V, Ghidoni S. Arrhythmias presenting in neonatal lupus. Scand J Immunol 2010;72:198-204.

6. Michaelsson M, Jonzon A, Riesenfeld T. Isolated congenital complete atrioventricular block in adult life. A prospective study. Circulation 1995;92:442-9.

7. Sonesson SE, Salomonsson S, Jacobsson LA, Bremme K, Wahren-Herlenius M. Signs of first-degree heart block occur in one-third of fetuses of pregnant women with anti-SSA/Ro 52-kd antibodies. Arthritis Rheum 2004;50:1253-61.

8. Theander E, Brucato A, Gudmundsson S, Salomonsson S, Wahren-Herlenius M, Manthorpe R. Primary Sjogren's syndrome--treatment of fetal incomplete atrioventricular block with dexamethasone. J Rheumatol 2001;28:373-6. 
9. Saleeb S, Copel J, Friedman D, Buyon JP. Comparison of treatment with fluorinated glucocorticoids to the natural history of autoantibody-associated congenital heart block: retrospective review of the research registry for neonatal lupus. Arthritis Rheum 1999;42:2335-45.

10. Hutter D, Silverman ED, Jaeggi ET. The benefits of transplacental treatment of isolated congenital complete heart block associated with maternal anti-Ro/SSA antibodies: a review. Scand J Immunol 2010;72:235-41.

11. Salomonsson S, Strandberg L. Autoantibodies associated with congenital heart block. Scand J Immunol 2010;72:185-8.

12. Julkunen H, Eronen M. Long-term outcome of mothers of children with isolated heart block in Finland. Arthritis Rheum 2001;44:647-52.

13. Julkunen H, Miettinen A, Walle TK, Chan EK, Eronen M. Autoimmune response in mothers of children with congenital and postnatally diagnosed isolated heart block: a population based study. J Rheumatol 2004;31:183-9.

14. Salomonsson S, Dorner T, Theander E, Bremme K, Larsson P, Wahren-Herlenius M. A serologic marker for fetal risk of congenital heart block. Arthritis Rheum 2002;46:1233-41.

15. Wahren-Herlenius M. Immunological contribution to the pathogenesis of congenital heart block. Scand J Immunol 2010;72:171-2.

16. Salomonsson S, Sonesson SE, Ottosson L, et al. Ro/SSA autoantibodies directly bind cardiomyocytes, disturb calcium homeostasis, and mediate congenital heart block. J Exp Med 2005;201:11-7. 
17. Wahren-Herlenius M, Sonesson SE. Specificity and effector mechanisms of autoantibodies in congenital heart block. Curr Opin Immunol 2006;18:690-6.

18. Strandberg L, Winqvist O, Sonesson SE, et al. Antibodies to amino acid 200-239 (p200) of Ro52 as serological markers for the risk of developing congenital heart block. Clin Exp Immunol 2008;154:30-7.

19. Clancy RM, Kapur RP, Molad Y, Askanase AD, Buyon JP. Immunohistologic evidence supports apoptosis, IgG deposition, and novel macrophage/fibroblast crosstalk in the pathologic cascade leading to congenital heart block. Arthritis Rheum 2004;50:173-82.

20. Hornberger LK, Al Rajaa N. Spectrum of cardiac involvement in neonatal lupus. Scand J Immunol 2010;72:189-97.

21. Villain E, Coastedoat-Chalumeau N, Marijon E, Boudjemline Y, Piette JC, Bonnet D. Presentation and prognosis of complete atrioventricular block in childhood, according to maternal antibody status. J Am Coll Cardiol 2006;48:1682-7.

22. Eronen M, Miettinen A, Walle TK, Chan EK, Julkunen H. Relationship of maternal autoimmune response to clinical manifestations in children with congenital complete heart block. Acta Paediatr 2004;93:803-9.

23. Smits JP, Veldkamp MW, Wilde AA. Mechanisms of inherited cardiac conduction disease. Europace 2005;7:122-37.

24. Brucato A, Franceschini F, Gasparini M, et al. Isolated congenital complete heart block: longterm outcome of mothers, maternal antibody specificity and immunogenetic background. J Rheumatol 1995;22:533-40. 
25. Dörner T, Chaoui R, Feist E, Goldner B, Yamamoto K, Hiepe F. Significantly increased maternal and fetal IgG autoantibody levels to $52 \mathrm{kD}$ Ro (SS-A) and La(SS-B) in complete congenital heart block. J Autoimmun 1995;8:675-84.

26. Silverman ED, Buyon J, Laxer RM, et al. Autoantibody response to the Ro/La particle may predict outcome in neonatal lupus erythematosus. Clin Exp Immunol 1995;100:499-505.

27. Buyon JP, Winchester RJ, Slade SG, et al. Identification of mothers at risk for congenital heart block and other neonatal lupus syndromes in their children. Comparison of enzyme-linked immunosorbent assay and immunoblot for measurement of anti-SSA/Ro and anti-SS-B/La antibodies. Arthritis Rheum 1993;36:1263-73.

28. Julkunen H, Kurki P, Kaaja R, et al. Isolated congenital heart block - Long-term outcome of mothers and characterization of the immune response to SS-A/Ro and to SSB/La. Arthritis Rheum 1993;36:1588-98.

29. Meilof JF, Frohn-Mulder IM, Stewart PA, et al. Maternal autoantibodies and congenital heart block: no evidence for the existence of a unique heart block-associated anti-Ro/SS-A autoantibody profile. Lupus 1993;2:239-46.

30. Dörner T, Feist E, Wagenmann A, et al. Anti-52 kDa Ro(SSA) autoantibodies in different autoimmune diseases preferentially recognize epitopes on the central region of the antigen. J Rheumatol 1996;23:462-8.

31. Buyon JP, Ben-Chetrit E, Karp S, et al. Acquired congenital heart block. Pattern of maternal antibody response to biochemically defined antigens of the SSA/Ro-SSB/La system in neonatal lupus. J Clin Invest 1989;84:627-34. 
32. Gordon P, Khamashta MA, Rosenthal E, et al. Anti-52 kDa Ro, anti-60 kDa Ro, and anti-La antibody profiles in neonatal lupus. J Rheumatol 2004;31:2480-7. 


\section{TABLE 1.}

Ro52, Ro60 and La serologic profile in antibody-positive mothers ( $n=93)$.

\begin{tabular}{lll}
\hline & Ro52 positive mothers & Ro52 negative m \\
& $\mathrm{n}(\%)$ & $\mathrm{n}(\%)$ \\
\hline Ro60+ / La+ & $43(49)$ & $1(20)$ \\
Ro60+ / La- & $14(16)$ & $2(40)$ \\
Ro60- / La+ & $9(10)$ & $1(20)$ \\
Ro60- / La- & $22(25)$ & $1 *(20)$ \\
\hline Total & $88(100)$ & $5(100)$ \\
\hline
\end{tabular}

*One Ro52/Ro60/La antibody-negative mother had only anti-histone antib 


\section{FIGURE LEGENDS}

Figure 1. An overview of patient population identification, resulting in blood sample collection from 193 mothers of children with AV block.

Figure 2. Autoimmune serology in mothers of children with AV block included in the study. In total, $48 \%(93 / 193)$ of the mothers analyzed demonstrated a positive autoantibody profile with antibodies against any of the antigens investigated.

Figure 3. Antibody profile in mothers with autoantibodies. (A) A majority, 95\% (88/93) of the mothers had antibodies against Ro52, while 63\% (59/93) and 58\% (54/93) demonstrated antibody-reactivity against the Ro60 and La proteins respectively. Antibody-reactivity outside the Ro/La complex was most frequently directed against histones, and anti-histone antibodies was demonstrated in $8 \%(7 / 93)$ of the mothers. (B) Occurrence of $\operatorname{IgA}$ and $\operatorname{IgM}$ anti-Ro52 antibodies in IgG anti-Ro52 positive mothers.

Figure 4. Antibodies against p200, a sequence within aa 200-239 of the Ro52 protein, analyzed by ELISA. Antibodies against p200 were detected in a majority of mothers carrying antibodies toward Ro52 (53/88, 60\%). The line indicates the mean (1.4) and the hatched line represents the cutoff for a negative/positive test ( 0.7 units). 
Figure 5. Correlation of maternal antibody levels to p200 and (A) time elapsed between delivery of child with AV block and blood sampling $(r=-0.40, p<0.0001)$ and $(B)$ maternal age at the time of blood sampling $(\mathrm{r}=-0.33, \mathrm{p}=0.0016)$, respectively. 


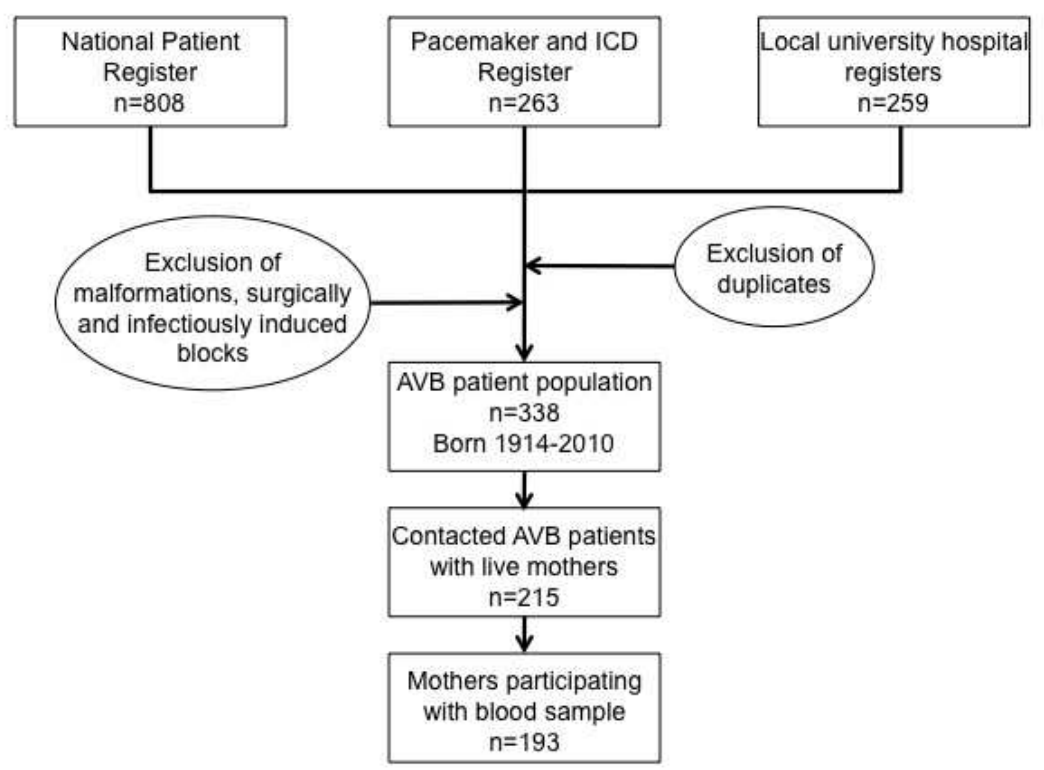

Figure 1, Salomonsson et al

$254 \times 190 \mathrm{~mm}(72 \times 72 \mathrm{DPI})$ 


1
2
3
4
5
6
7
8
9
10
11
12
13
14
15
16
17
18
19
20
21
22
23
24
25
26
27
28
29
30
31
32
33
34
35
36
37
38
39
40
41
42
43
44
45
46
47
48
49
50
51
52
53
54
55
56
57
59
60

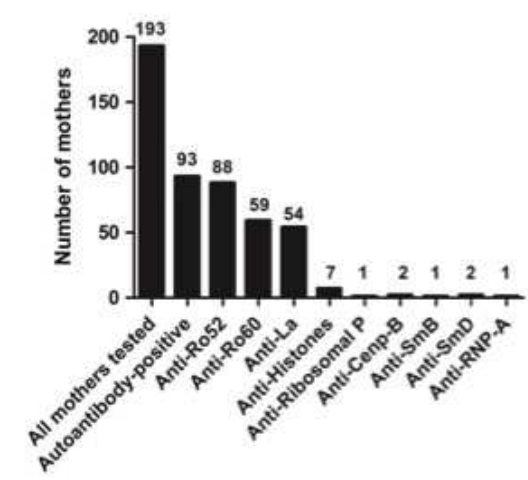

Figure 2, Salomonsson et al

$254 \times 190 \mathrm{~mm}(72 \times 72 \mathrm{DPI})$ 


1
2
3
4
5
6
7
8
9
10
11
12
13
14
15
16
17
18
19
20
21
22
23
24
25
26
27
28
29
30
31
32
33
34
35
36
37
38
39
40
41
42
43
44
45
46
47
48
49
50
51
52
53
54
55
56
57
58
60

A

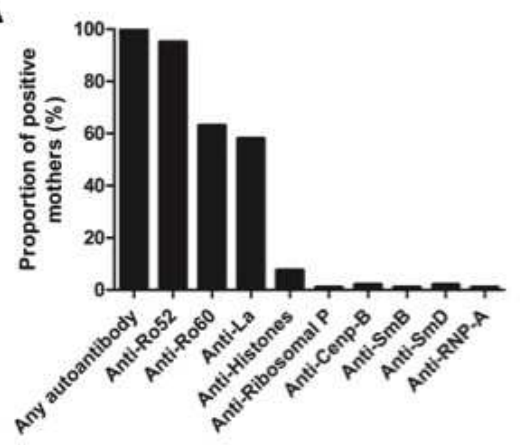

B

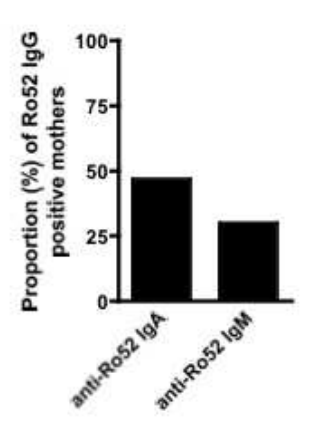

Figure 3, Salomonsson et al

$254 \times 190 \mathrm{~mm}(72 \times 72 \mathrm{DPI})$

Scandinavian Journal of Immunology 


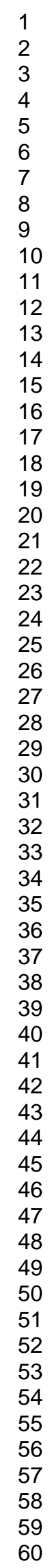

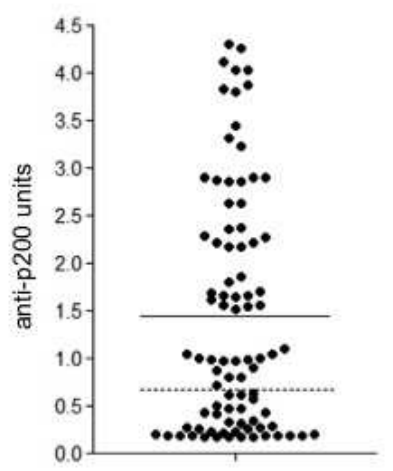

Figure 4, Salomonsson et al

$254 \times 190 \mathrm{~mm}(72 \times 72$ DPI $)$

Scandinavian Journal of Immunology 
1

2

3

4

5

6

7

8

9

10

11

12

13

14

15

16

17

18

19

20

21

22

23

24

25

26

27

28

29

30

31

32

33

34

35

36

37

38

39

40

41

42

43

44

45

46

47

48

49

50

51

52

53

54

55

56

57

58

59

60
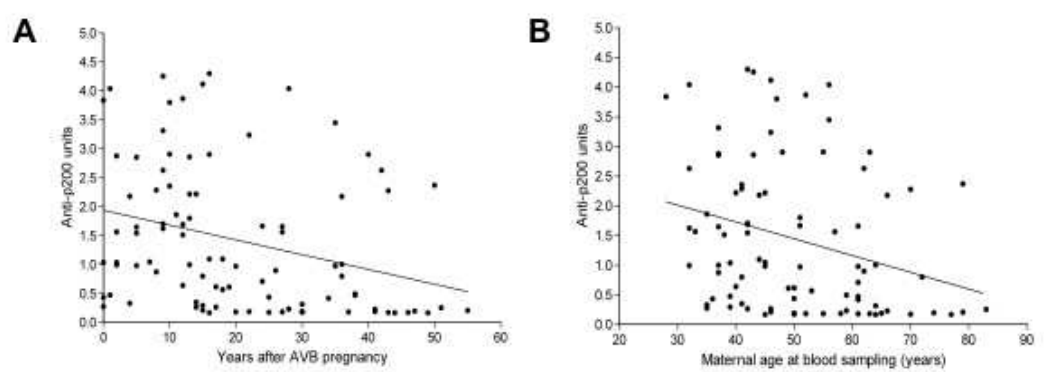

Figure 5, Salomonsson et al

$254 \times 190 \mathrm{~mm}(72 \times 72 \mathrm{DPI})$

Scandinavian Journal of Immunology 